\title{
Lessons learned during construction and test of the GLAST Large Area Telescope Tracker
}

\author{
Luca Latronico on behalf of the LAT Tracker team \\ INFN-Pisa, Via Buonarroti 2, 56100 Pisa, Italy
}

\begin{abstract}
The GLAST Large Area Telescope (LAT) is a satellite gamma-ray observatory designed to explore the sky in the energy range $20 \mathrm{MeV} \simeq 300 \mathrm{GeV}$, a region populated by emissions from the most energetic and mysterious objects in the cosmos, like black holes, AGNs, supernovae, gamma-ray bursters. The silicon-strip tracker is the heart of the photon detection system, and with its $80 \mathrm{~m}^{2}$ of surface and almost $1 \mathrm{M}$ channels is one of the largest silicon tracker ever built. Its construction, to be completed by 2006, and the stringent requirements from operation in space, represent a major technological challenge. Critical design, technology and system engineering issues are addressed in this paper, as well as the approach being followed during construction, test and qualification of the LAT silicon tracker.
\end{abstract}

Key words: gamma-ray, satellite, pair-conversion, silicon, strip, tracker PACS: 95.55.-n, 95.55.K, 95.85.P, 29.40.Gx

\section{The GLAST mission}

The GLAST mission has a vast and ambitious science program, which involves observations of gamma-rays emitted in a broad energy window from a wide range of cosmological sources, from steady state sources like Active Galactic Nuclei, Super Novae Remnants, black-holes to transients phenomena like Gamma Ray Bursts and flares. Previous missions studying this high energy photon regime, like EGRET ([1]), provided unmistakable evidence of an incredibly vast and rich phenomenology, but potential discoveries were limited by a conservative design of the instrument.

The design and the choice of technologies for the Large Area Telescope (LAT), the main instrument onboard GLAST, were inspired by the desire to improve sensitivity and resolution to the best achievable performance, the final goal of the mission being the measurement of direction, energy and arrival time of 
photons from the whole sky with the most accurate resolution. This objective has to be met given the typical constraints of space applications like limitations on mass, power, consumables and specific requirements of system redundancy and reliability.

The LAT implements the same EGRET concept of the pair-conversion telescope, but application of state-of-the-art detection technologies allow an improvement of more than an order of magnitude in resolution and sensitivity. Gamma-rays crossing GLAST convert into $e^{+} / e^{-}$pairs in a high resolution

tracker-converter, a sequence of silicon-strip detectors and tungsten layers. The electrons energies are measured in a CsI, imaging calorimeter placed downstream the tracker. Rejection of charged particles background is done with an external, segmented anti-coincidence shield built with plastic scintillators and operating at high detection efficiency $(>0.9997)$ over the whole LAT surface $([2])$.

\section{Construction of the LAT Tracker and lessons learned}

Redundancy is a key factor in space missions, protecting from single-point failures and adding considerable reproducibility to the production, with obvious benefits on quality and costs. For this reason, the LAT design was implemented with high modularity at all levels, and the telescope is itself an array of $4 \times 4$ identical towers, each equipped with a tracker, a calorimeter and its own data acquisition module, all installed on a mechanical grid integrated onto the satellite. The tracker towers are also built from identical hardware structures, called trays, which integrate the silicon detectors, the front-end electronics, the supporting mechanics and tungsten conversion layers of various thicknesses. Each tower is then assembled with 17 standard type trays and a special top and bottom trays, which are stacked with orientation alternating at $90^{\circ}$ to provide 18 xy detection layer. The read-out architecture has built-in redundancy, using double paths for data, control and trigger lines (see [3] for a detailed description).

A major improvement towards higher sensitivity was the instrumentation of a large area tracking surface $\left(80 \mathrm{~m}^{2}\right)$. The technological development which allow building such large tracking systems is the production reliability of large area, single-sided, AC-coupled silicon strip detectors (SSD) from 6 inches wafers. These sensors are the basic building blocks of the LAT tracker, and more than $10 \mathrm{~K}$ such detectors are assembled into larger area detection units for its construction. Use of silicon detectors, besides the obvious advantage of using no consumables, offers very attractive improvements to the tracker performance, like a short instrumental dead-time $(\sim 20 \mu \mathrm{s})$, due to fast signal shaping and absence of recovery time, and high spatial resolution over a large geometrical 
acceptance. In fact thin detection layers can be densely stacked in height and favour the detector aspect ratio, so that tracks entering the detector from a large field of view are reconstructed using many high resolution hits.

The total power budget allocated for the GLAST tracker is 160W. Aggressive specifications for the sensors leakage current and depletion voltage were therefore requested to Hamamatsu Photonics, the company providing the silicon detectors, in order to minimize power consumption. The contribution of the sensors to the tracker power consumption will not exceed few watts even after an irradiation ten times higher than the expected integrated dose in 5 years operation([5]). The whole production of 11500 SSDs was procured and tested at INFN laboratories, with an overall rejection rate of $\simeq 0.5 \%$. Table 1 summarizes some of the excellent test results obtained, while detailed description of the sensors and the tests can be found in [4].

The second step in the construction of the LAT tracker is ladder assembly: four SSDs are glued head to head, their strips are wire-bonded in order to obtain $40 \mathrm{~cm}$ long strips and bondings are encapsulated. The number of ladders to produce is large ( $>2500)$, and their assembly requires high levels of quality for a sequence of delicate operations like gluing, micro-bonding of strips, encapsulation of bonds. For this reason two high-tech italian companies ( $G \& A$ Engineering, Mipot) were qualified for engineering ladder assembly in close collaboration with INFN laboratories and finally assemble flight ladders. Quality assurance for ladder production is based on electrical and alignment tests performed by INFN personnel at the production sites. Global tests of leakage current, bulk capacitance, depletion voltage are performed on each ladder, and single strip tests check integrity of the strips after bonding and encapsulation by measuring the strip current and coupling capacitance. Alignment of the sensors along the ladder is also measured. Results from the production of the first 1000 flight ladders are summarized in table 1 . The overall rejection rate of flight ladders was $\simeq 1.5 \%$. Measurements on the ladders are very well in agreement with what expected from the composing SSDs: the leakage current measured after encapsulation is only slightly higher than the sum of the SSDs and the depletion voltage is exactly the same; the distribution of alignments has an RMS of only $2 \mu \mathrm{m}$, the same spread of the SSD wafer cut distribution, indicating that the alignment and gluing procedure, which simply rely on referencing jigs and makes no use of expensive and lengthy CMM positioning, maintains the already very accurate alignment of the Hamamatsu dicing.

The same approach based on a close collaboration between scientific institutions and high-tech companies was followed for development of the mechanics supporting the live sensors. Trays are composite panels built around an $\mathrm{Al}$ honeycomb core with carbon fiber face-sheets and kapton bias circuit for the top and bottom sides, where silicon ladders are glued, and four side carboncarbon close-outs supporting the read-out electronics hybrid and the structure 
Table 1

Silicon sensors test results summary

\begin{tabular}{|c|c|c|c|c|c|}
\hline Tested device & \multicolumn{3}{|c|}{ all SSDs(11500) } & \multicolumn{2}{|c|}{ 1000-Ladders } \\
\hline Measurement & average & RMS & specs & average & RMS \\
\hline$\overline{\text { Leakage }_{\text {current }}{ }^{1}(\mathrm{nA})}$ & 110 & 40 & $<200$ & 580 & 180 \\
\hline Depletion voltage (V) & 67 & 18 & $<120$ & 63 & 15 \\
\hline Bulk capacitance $^{1}(\mathrm{pF})$ & 1830 & 10 & - & 7500 & 50 \\
\hline Wafer alignment $(\mu \mathrm{m})$ & 0 & 2 & $<20$ & 0 & 2 \\
\hline Broken strips (overall rate) & $10^{-4}$ & - & $<10^{-2}$ & $3 \cdot 10^{-4}$ & - \\
\hline
\end{tabular}

${ }^{1}$ measured at $150 \mathrm{~V}$ bias

itself. Fig. 1 shows an exploded view of the tray structure. Trays mechanics are manufactured at Plyform, an italian company specialized in assembly of composite materials, and their quality is checked after all significant production steps, in order to minimize the risk of expensive rejection of parts that are well ahead in the assembly cycle. A non-destructive interferometric test is performed on the bare panel for search of resonance frequencies and assembly defects, as well as a thermal-vacuum $\left(55^{0} \mathrm{C}, 10^{-4} \mathrm{bar}\right)$ and a vibrational $(20-2000 \mathrm{~Hz})$ cycle for space qualification. Integration of tray mechanics, silicon sensors and front-end electronics is again performed in industry (G\&A), under careful supervision of INFN representatives. The complete qualification of trays is done inside INFN laboratories, going through functional tests, a thermal-cycle and a cosmic rays burn-in test. Trays are finally assembled into towers, whose performance is extensively tested with cosmic rays. Before delivery to SLAC for the final integration with the others subsystems and the grid, tracker towers are qualified for flight with thermal-vacuum and vibrational tests performed in Alenia Spazio by an INFN team and the local company team operating the facility.

Given the complexity of the tracker production and the many institutions involved, an efficient management of the project is mandatory. Particularly important was a clear and well-defined assignment of goals and responsibilities to the collaborating institutions, so that each site has the necessary freedom to choose comfortable solutions to achieve the given task, such as choice of suppliers, service software, database systems and hardware instrumentation. On the other hand efficient communication and data transfer between the many sites and industrial partners involved needs to be guaranteed, and common protocols and agreed procedures must be used. A strong quality program was therefore put in place in all scientific institutions of the collaboration, and tight quality control over all activities was inforced. Working with a quasicommercial attitude, tracking of all production and test activities is recorded with the aid of a custom database system that implements tools for incoming of single components or composite parts, data storage and analysis, parts selection, workflow tracking, test validation and tracking, nonconformance reporting. Quality assurance in the activities of the industrial suppliers rely on space industry standards. Figure 2 shows as an example the manufacturing 


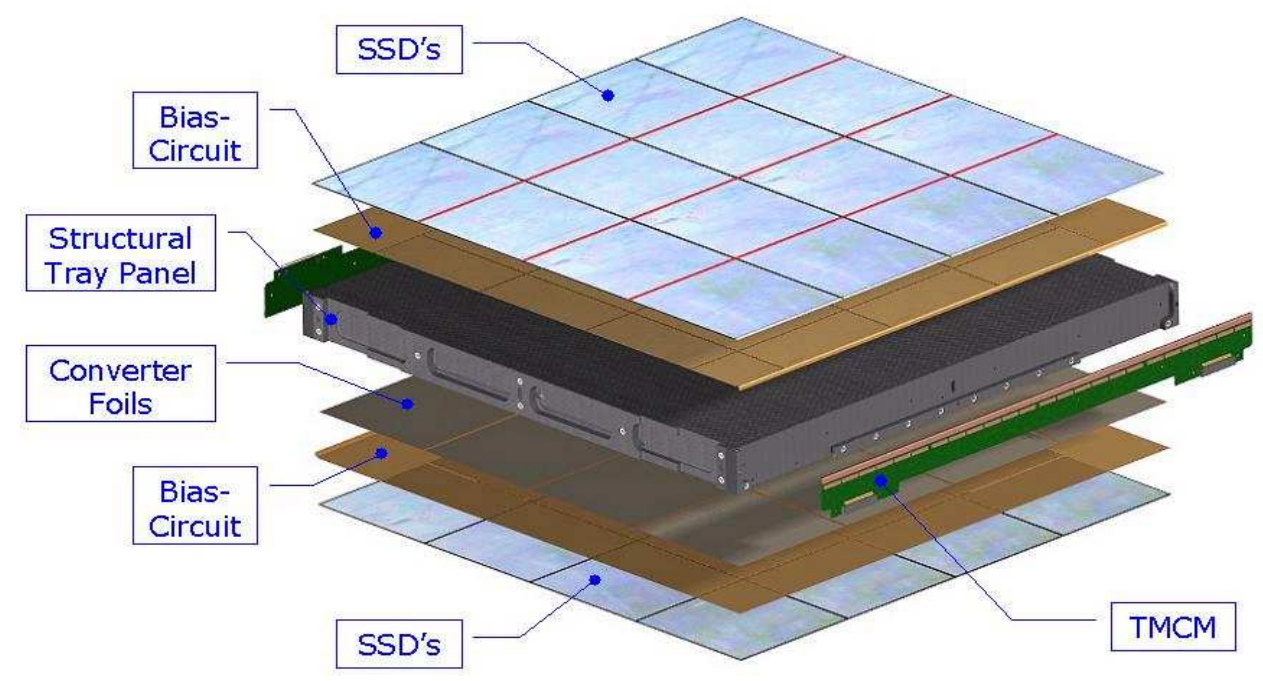

Fig. 1. Exploded view of a tray and its subcomponents

and test flowchart of a flight tray as recorded in the central data server at INFN-Pisa, where the information flow from all the different sources.

A collaborative relationship with high-technology companies proved to be essential for the project, for the procurement of excellent silicon sensors, reliable high-quality assemblies of ladders and trays and access to facilities for environmental tests and space qualification. In dealing with the many industrial partners, direct contracts between the scientific institution and the companies allowed a constant control over production from project people, by means of onsite inspection and supervision. This is very important for early detection of failures and prompt corrective actions. On the other hand, an interaction with a main contractor which subcontracts procurement of key parts to other companies has several disadvantages, particularly when manufacturing highly customized hardware parts. A main contractor will be forced to require heavy financial overheads to cover expenses for insurances and deposits requested by subcontractors. On the technical side, the main contractor will tend to minimize its own risk by overconstraining the parts supplied by subcontractors requiring very tight tolerances, with negative consequences on costs and schedule. Finally, technical supervision from project people would need to be agreed with the main contractor, often resulting in a delayed feedback.

The first model was successfully applied by INFN for construction of two tracker engineering model prototypes, and production of the first flight parts. A full-size mechanical tower, built using flight trays equipped with fake silicon 


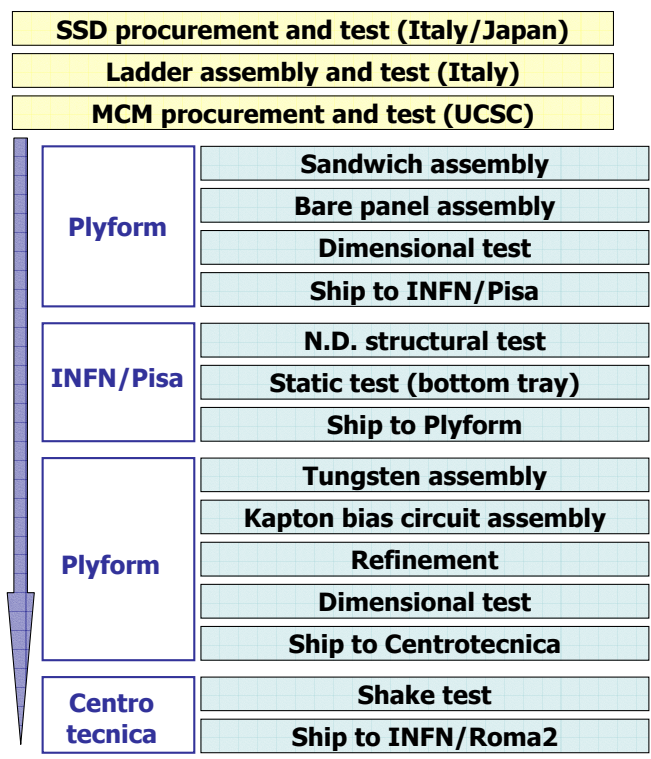

\begin{tabular}{|c|c|}
\hline \multirow{2}{*}{ INFN/Roma2 } & Thermal-vacuum test \\
\hline & Ship to G\&A \\
\hline \multirow{7}{*}{$\begin{array}{c}\text { G\&A } \\
\text { Engineering }\end{array}$} & MCM assembly \\
\hline & Ladder gluing \\
\hline & Ladder bonding \\
\hline & Encapsulation \\
\hline & Mechanical test \\
\hline & Electrical test \\
\hline & Ship to INFN \\
\hline \multirow{5}{*}{$\begin{array}{l}\text { INFN/Bari } \\
\text { INFN/Perugia } \\
\text { INFN/Pisa } \\
\text { INFN/Roma2 }\end{array}$} & Tray incoming test \\
\hline & Thermal cycle \\
\hline & Functional test \\
\hline & C.R. burn-in and functional test \\
\hline & Ship to INFN/Pisa \\
\hline \multicolumn{2}{|r|}{ Tower assembly (INFN/Pisa) } \\
\hline \multicolumn{2}{|c|}{ Towers integration and test (SLAC) } \\
\hline
\end{tabular}

Fig. 2. LAT tray manufacturing and test flow-chart

and electronics, had a final alignment smaller than $100 \mu \mathrm{m}$, i.e. less than $1 / 3$ the required tolerance, and survived full-scale thermal-balance and vibrational test cycles. A mini-tracker tower, equipped with 5 live flight trays, met the performace requirements of a hit detection efficiency $>99 \%$ up to $1 / 2 \mathrm{MIP}$ threshold, and a single strip noise occupancy $<10^{-6}$ already at a threshold of $1 / 4 \mathrm{MIP}$.

\section{Conclusions}

The GLAST project is a powerful partnership between the HEP and Astrophysics communities, bringing together complimentary expertise for the construction and operation of a very advanced satellite gamma-ray telescope 
capable of answering their common scientific objectives. The superior sensitivity, angular and energy resolution that will enable the LAT to meet this goal are obtained through a careful design and use of leading-edge particle detectors. Particularly challenging is the construction of the LAT silicon tracker, the largest ever built for space applications, which required a close collaboration with qualified industrial partners for production of high-standards flight parts and efficient system engineering. Tight quality assurance controls throughout all phases of the tracker assembly, similar to industrial standards, proved to be essential for assembling preliminary prototypes with performances outstanding specifications, and will be crucial for commissioning the instrument on schedule for the early 2007 launch by NASA.

\section{References}

[1] P. Nolan et al., IEEE Trans. Nucl. Sci., 39, 993-996, 1992

[2] E. Bloom et al., Proposal for the Gamma-ray Large Area Space Telescope, SLAC-R-22, Feb. 1998 - see also http://glastserver.pi.infn.it/glast

[3] M. Sugizaki, these Proceedings

[4] T. Ohsugi, these Proceedings. R. Bellazzini et al., Nucl. Instr. and Meth. A523, issues $1-1$ (2003) 136

[5] S. Yohsida et al., IEEE Transactions, Volume 49, Issue: 3, June 2002 Pages:1017 $-1021$ 